\title{
Remembering and forgetting: directed forgetting effect in obsessive-compulsive disorder
}

\author{
This article was published in the following Dove Press journal: \\ Neuropsychiatric Disease and Treatment \\ 14 June 201 I \\ Number of times this article has been viewed
}

\section{Mika Konishi' \\ Kurie Shishikura ${ }^{2}$ \\ Shutaro Nakaaki ${ }^{3}$ \\ Shin-ichi Komatsu ${ }^{4}$ \\ Masaru Mimura' \\ 'Department of Neuropsychiatry, Keio University School of Medicine, Tokyo; ${ }^{2}$ Department of Neuropsychiatry, Kitasato University School of Medicine, Kanagawa; ${ }^{3}$ Department of Psychiatry and Cognitive-Behavioral Medicine, Nagoya City University Graduate School of Medical Science, Nagoya; ${ }^{4}$ Faculty of Education, Shinshu University, Nagano, Japan}

Correspondence: Mika Konishi Department of Neuropsychiatry, Keio University School of Medicine, 35 Shinanomachi, Shinjuku-ku, Tokyo 160-8582, Japan

Tel +81353633829

Fax +81353790187

Email mikita1204@ymail.plala.or.jp

\begin{abstract}
It has been reported that episodic memory seems to be impaired in patients with obsessive-compulsive disorder (OCD) because the patients repeat a specific checking behavior, but it is still unknown if OCD patients show memory impairments associated with their unique symptoms or not. To study episodic memory in OCD patients, we examined the directed forgetting effect. Patients with OCD and healthy control participants were given a list of 24 emotionally neutral everyday words (12 remember [R]-cued words and 12 forget [F]-cued words) under two conditions: List and Item. The results of our study showed that OCD patients recalled a number of F-cued words similar to that for controls and relatively fewer R-cued words than controls under both List and Item conditions. Consequently, the directed forgetting effect was smaller in OCD patients than controls. Our results demonstrated that both selective encoding and retrieval inhibition processes are impaired in OCD, and we suggest that recall of unfavorable items to be forgotten intruded into necessary items to be remembered. This impairment in episodic memory may partially account for some of the unique clinical symptoms of OCD.
\end{abstract}

Keywords: episodic memory, retrieval inhibition, selective encoding

\section{Introduction}

Patients with obsessive-compulsive disorder (OCD) show unique symptoms such as specific intrusive thoughts, associated with repetitive behaviors aimed at removing the unwanted thoughts. OCD patients are stressed from expelling the obsessive thoughts from their minds and can report in detail what they have done to rid themselves of these irrational thoughts. Such clinical observations may tell us that OCD patients appear to exhibit sufficient memory for a specific event. On the other hand, episodic memory in relation to checking behaviors seems to be deficient in OCD patients because they repeat the checking behaviors. ${ }^{1-5}$

Debate continues as to whether or not OCD patients exhibit memory impairments. ${ }^{6-8}$ One of the most consistent findings in previous literature has been the impairment of nonverbal memory. ${ }^{9-14}$ Most studies of memory in OCD focused on weakened memory representation rather than poor memory performance itself, resulting in the assumption that a decline in meta-memory, such as an impairment in memory representation of one's own behavior, would bring about the obsessive thoughts and repetitive behaviors. ${ }^{1,4,15}$ In contrast, other studies have proposed an OCD memory model in which the patient is challenged to explain memory distrust in spite of repetitive checking. ${ }^{2,3}$ These experiments, in which control subjects were required to check a virtual gas stove in a computer animation, confirmed that repeated checking behavior contributes directly to weakening of self-confidence concerning the memory trace 
and to decline in meta-memory even though actual memory accuracy is unaltered.

A different view holds that poor memory recall in OCD patients is a secondary deficit reflecting impaired organization strategies at the stage of encoding stimuli; this underlying executive dysfunction is considered the core deficit affecting visual and verbal memory. ${ }^{16-20}$ Thus, these studies concluded that cognitive characteristics of OCD are related to dysfunction of the frontal-subcortical system in their neurobiological models of OCD.

Taken together, the previous reports have suggested various memory deficits in OCD without providing a consensus view of neuropsychological evidence of memory deficits specific to OCD that contribute to its unique symptoms. In formulating the present investigation, we assumed existence of a deficit affecting a normal forgetting process by which persons forget irrelevant material for the sake of remembering relevant material, in relation to episodic memory in OCD. In other words, we assumed that OCD patients fail to forget what they do not need to remember with the result that they fail to remember what they need to remember. To examine episodic memory with respect to the clinical features of OCD, we selected a method called a "directed forgetting paradigm" 21 in which subjects given a considerable number of words to learn are told to intentionally forget some of what they have learned.

While unintentional forgetting can be described as a failure to remember, intentional forgetting seems to require a strategic mechanism in memory that functions to reduce interference in processing and retrieval of relevant materials. The specific mechanism that accomplishes successful intentional forgetting depends on whether or not the material has been encoded into long-term memory by the time the instruction to forget is given. On the one hand, when a group of items is presented followed by an instruction either to remember or to forget, the items already have been committed to longterm memory; the encoding process is the same for either instruction. Consequently, the instruction to forget is likely to cause the representation of the material to be suppressed or inhibited in subsequent retrieval attempts. Such retrieval inhibition is believed to occur in a list-method directed forgetting paradigm. If, on the other hand, the instruction to forget or remember is presented following each item; the instruction to forget can prevent commitment to long-term memory. In this case, forgetting does not operate upon a stored representation but upon the processes that otherwise would lead to long-term storage. Items to be forgotten would be suppressed or inhibited at the point of encoding, such selective encoding is considered a key factor in item-method directed forgetting.

Previous studies in healthy persons support the method-based theoretical distinction, comparing with itemmethod directed forgetting, showing that performance in list-method directed forgetting increases recall of items to be forgotten while there is no difference in the recall of items to be remembered. ${ }^{22,23}$ The directed forgetting effect permits a quantitative index of the ability to suppress irrelevant material to permit successful recall of relevant material, represents the difference between the number of to-be-remembered words and the number of to-be-forgotten words that are recalled. Healthy persons generally are assumed to benefit from a large directed forgetting effect since the instruction to remember facilitates retention while the instruction to forget prompts intentional forgetting.

We believe that directed forgetting is a particularly informative method for examining the characteristics of episodic memory in OCD. Our hypothesis in the present investigation was that OCD patients would recall more items to be forgotten than healthy persons whereas OCD patients would recall fewer items to be remembered than healthy persons. Consequently, we hypothesize that the directed forgetting effect would be much smaller in OCD patients than in healthy persons. In addition, we assumed that OCD patients would be impaired in either retrieval inhibition or selective encoding if a smaller directed forgetting effect in OCD was observed either in list- or item-method, respectively. If the directed forgetting effect was smaller in OCD patients than in healthy persons both in list- and item-methods, then it is assumed that OCD patients are impaired in both retrieval and encoding phases. All previous studies applying the directed forgetting paradigm to OCD patients have shown a decreased directed forgetting effect in either free recall or a recognition test, ${ }^{24-26}$ that was limited to negative or OCD-related words. Moreover, each study used only a list-based or an item-based method in the directed forgetting paradigm. In the present study, we used both list and item methods for a directed forgetting paradigm that included a list of neutral nouns aiming to explore both encoding and retrieval processes in episodic memory.

\section{Methods}

\section{Participants}

Twenty-eight OCD patients (14 males and 14 females, ages 16 to 68 years) were recruited from Showa University Hospital. These participants were all found to meet DSM-IV ${ }^{27}$ criteria for a diagnosis of OCD as determined by 
structured clinical interviews (Structured Clinical Interview for DSM-IV, Clinical Version, SCID/CV). ${ }^{28}$ Their mean score for the total scale of the Yale-Brown Obsessive-Compulsive Scale (Y-BOCS) ${ }^{29}$ was $22.4 \pm 5.2$ (total score of obsession; $11.1 \pm 2.9$, total score of compulsion; $11.3 \pm 2.6$ ), indicating moderate severity. Exclusion criteria were as follows; current major depression or substance abuse, or any history of a psychotic episode, substance dependence, head injury, or neurologic disorder (including Tourette's syndrome). In addition, patients with axis II disorders were excluded. All diagnoses were confirmed by the second author (KS, a licensed senior psychiatrist). None of the patients had received cognitive behavior therapy, but all patients were taking a psychotropic medication such as fluvoxamine or paroxetine at the time of neuropsychological tests. The equivalence of each drug was calculated according to a previously described method ${ }^{30}$ in which the recommended therapeutic dose of clomipramine was $150 \mathrm{mg} /$ day. The mean patient age was $36.4(\mathrm{SD}=14.0)$, and the mean duration of symptoms was 9.6 years $(\mathrm{SD}=9.8)$.

Seventeen healthy control participants (ten males and seven females) took part in this study as volunteers. They were not taking any psychoactive medications and were free of psychiatric disorders (as verified by SCID interview), neurological disorders, or other significant medical illnesses. Their ages ranged from 24 to 58 years (mean age $=32.7 \pm 10.3$ years $)$, and they did not differ from the OCD patients with regard to age $(\mathrm{t}[43]=-1.44, P>0.05)$ and gender $\left(\chi^{2}=0.33, P>0.05\right)$.

OCD patients were not impaired in general intelligence (Wechsler Adult Intelligence Scale-Revised), conventional word list learning (Rey Auditory-Verbal Learning Test), or frontal-executive functions (Modified Stroop Test, Word List Generation, and Wisconsin Card Sorting Test [Keio version]) (all $P>0.05$ ), compared with our healthy volunteers database of 28 persons age- and gender-matched to the OCD patients (mean age $=37.5 \pm 14.1 ; 14$ males and 14 females). Neuropsychological test profile of the OCD group and the healthy controls are shown in Table 1.

After complete explanation of the study, written informed consent was obtained from each participant, and the study was approved by the Ethics Committee of Showa University, School of Medicine, Tokyo, Japan.

\section{Materials}

Experimental stimuli in the directed forgetting paradigm included eight sets of 24 neutral nouns for two conditions; List and Item. A Macintosh desktop computer running
Table I Performance on neuropsychological tests in healthy controls $(n=28)$ and OCD patients $(n=28)$

\begin{tabular}{|c|c|c|}
\hline Test & $\begin{array}{l}\text { Healthy } \\
\text { controls }\end{array}$ & $\begin{array}{l}\text { OCD } \\
\text { patients }\end{array}$ \\
\hline WAIS-R Full-scale IQ & $101.3(9.2)$ & $93.2(11.0)$ \\
\hline \multicolumn{3}{|l|}{ Rey Auditory-Verbal Learning Test } \\
\hline $\begin{array}{l}\text { Immediate recall } \\
(\max =15)\end{array}$ & I3.I (2.2) & II.7 (2.2) \\
\hline $\begin{array}{l}\text { Delayed recall } \\
(\max =15)\end{array}$ & $1 \mathrm{I} .4(3.7)$ & $9.5(2.9)$ \\
\hline $\begin{array}{l}\text { Recognition } \\
(\max =15)\end{array}$ & I4.I (3.0) & $14.4(1.2)$ \\
\hline \multicolumn{3}{|l|}{ Modified Stroop test } \\
\hline $\begin{array}{l}\text { Part I (congruent } \\
\text { condition) (seconds) }\end{array}$ & $14.8(3.7)$ & $17.0(7.1)$ \\
\hline $\begin{array}{l}\text { Part III (incongruent } \\
\text { condition) (seconds) }\end{array}$ & $23.5(18.2)$ & $24.7(11.7)$ \\
\hline \multicolumn{3}{|l|}{ Word List Generation } \\
\hline Initial letter/minute & $8.6(3.5)$ & $8.0(2.6)$ \\
\hline Category/minute & $14.3(4.0)$ & $12.4(2.5)$ \\
\hline \multicolumn{3}{|c|}{ Wisconsin Card Sorting Test (Keio Version) } \\
\hline Categories achieved & $4.3(1.9)$ & $4.7(1.9)$ \\
\hline $\begin{array}{l}\text { Number of perseverative } \\
\text { errors }\end{array}$ & $2.5(3.1)$ & $2.3(4.8)$ \\
\hline
\end{tabular}

Notes: Data are expressed as group mean and standard deviations are in parentheses.

Abbreviations: OCD, obsessive compulsive disorder; WAIS-R, Wechsler Adult Intelligence Scale-Revised.

HyperCard software was used to present written instructions and stimulus words. Stimulus words were selected and were controlled with regard to the length of the words, commonness and emotional valence. ${ }^{31}$ Each word was written in Japanese kana characters and was presented visually and auditorily for 5 seconds (Figure 1). In the List condition, after half of a list of words were presented, written instructions appeared to tell the participant that these words should be forgotten, and the rest of the list was given as long as participants understood the instructions. In the Item condition, participants were given a list of words, and presentation of each word was followed by either $\circ$ or $\times$ to indicate an instruction to remember (R-cued word) or to forget (F-cued word), respectively. This instruction was displayed for 1 second, following an interstimulus interval of 1 second.

\section{Design}

There were two cue types (Remember and Forget), each in two separate task conditions (List and Item). Stimulus words were randomly selected with regard to cue types and task condition for each participant.

Within each task condition, numbers of correctly recalled words were recorded, and relative directed forgetting effect 


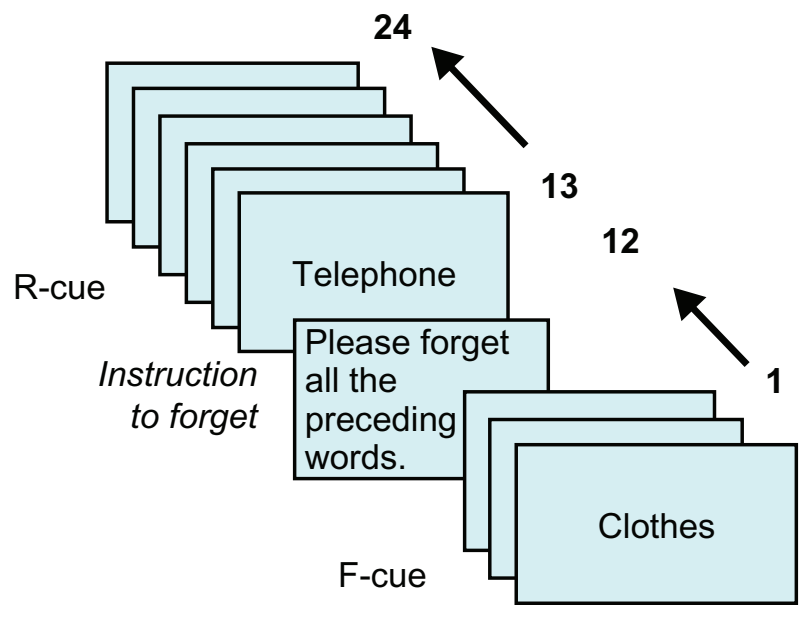

List condition

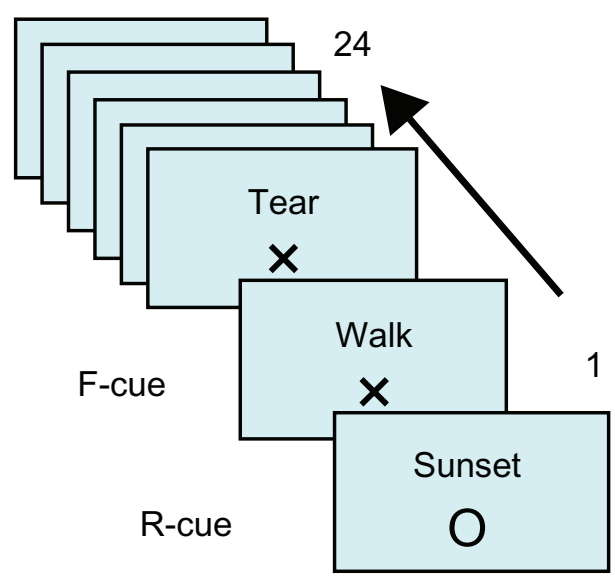

Item condition

Figure I Illustration of List and Item conditions in the directed forgetting paradigm.

was computed by subtracting the number of F-cued words recalled from the number of R-cued words recalled. Larger differences in scores indicated a greater directed forgetting effect.

A 2 (Group: Control, OCD) $\times 2$ (Task condition: List, Item) $\times 2$ (Cue: Remember, Forget) factorial design was employed. The first factor was measured between subjects, while the last two factors were measured within subject groups. For determining directed forgetting effects, a 2 (Group: Control, OCD) $\times 2$ (Task condition: List, Item) mixed design was employed.

\section{Procedure}

Initially (List condition), participants were told explicitly to learn as many words as possible, each of which would be presented one at a time on the computer screen. After half of the list had been shown in this way, instructions appeared, stating "What you have done thus so far has been practice. Therefore, you should forget all of the words that you have seen and heard so far". After participants were told to forget the remembered words, they were given the rest of the list to remember. Next, in the Item condition, participants were presented a list of words with each followed by a cue to either remember or forget. After presentation of words under each condition, participants were asked to recall the words they could remember, regardless of whether the original instruction said to remember or to forget each word. Participants were given 5 minutes or more until they indicated that they could recall no additional words. Participants were tested individually in a sound-attenuated room over the course of 30 minutes.

\section{Results}

\section{Task conditions in the directed forgetting paradigm}

Before analysis, the data for the control group were tested for any significant effect of task condition (List or Item). Two-tailed $t$-test showed a significant effect of task condition, and so the control group recalled more F-cued words in List condition than in Item condition $(P<0.01)$ (Cohen's $d s=1.33)$ whereas there was no difference in the recall of R-cued words with regard to task conditions $(P=0.94)$ $(d s=0.02)$. Likewise, OCD patients recalled more F-cued words in List condition than in Item condition $(P<0.01)$ $(d s=0.69)$, without difference in numbers of R-cued words recalled between List and Item conditions $(P=0.08)$ $(d s=0.29)$.

\section{Directed forgetting paradigm in OCD}

Mean numbers of correctly recalled words in List and Item conditions are presented in Figure 2. The number of correctly recalled words was then evaluated by analysis of variance (ANOVA) procedures using a three variable (Group $\times$ Task $\times$ Cue) mixed model, with repeated measures applied to Task and Cue. Analysis found significant main effects for group $(\mathrm{F}[1,42]=8.77, P<0.01)$, task $(\mathrm{F}[1,42]=22.65, P<0.05)$, and cue $(\mathrm{F}[1,42]=130.63$, $P<0.05)$. Most importantly, the Group $\times$ Cue interaction $(\mathrm{F}[1,42]=6.56, P<0.05)$ was significant, indicating that the OCD group recalled a smaller number of R-cued words than the control group under both List and Item conditions, while no group difference was evident in the number of F-cued words recalled under either condition. Thus, the mean 


\section{List condition}

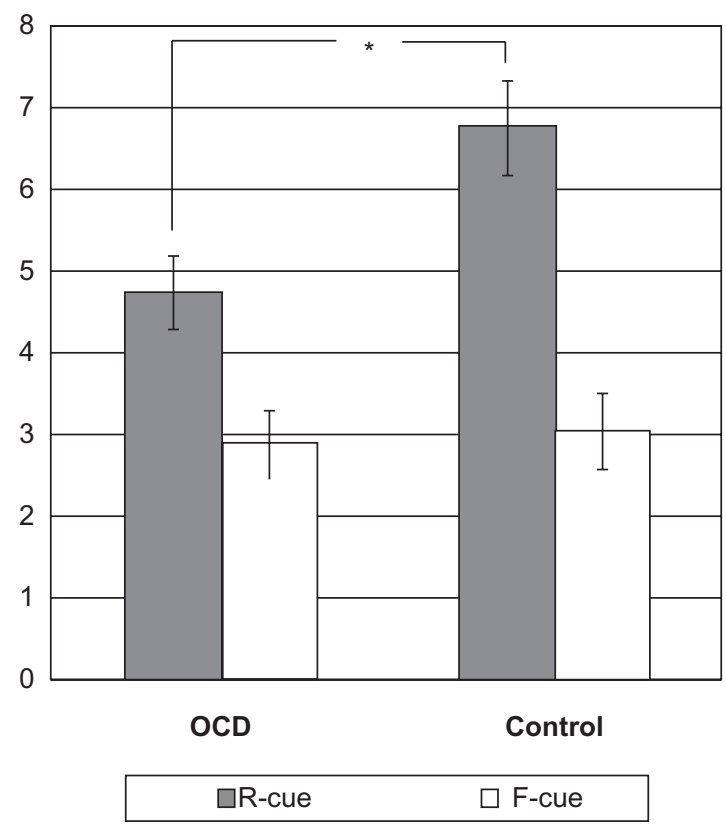

Item condition

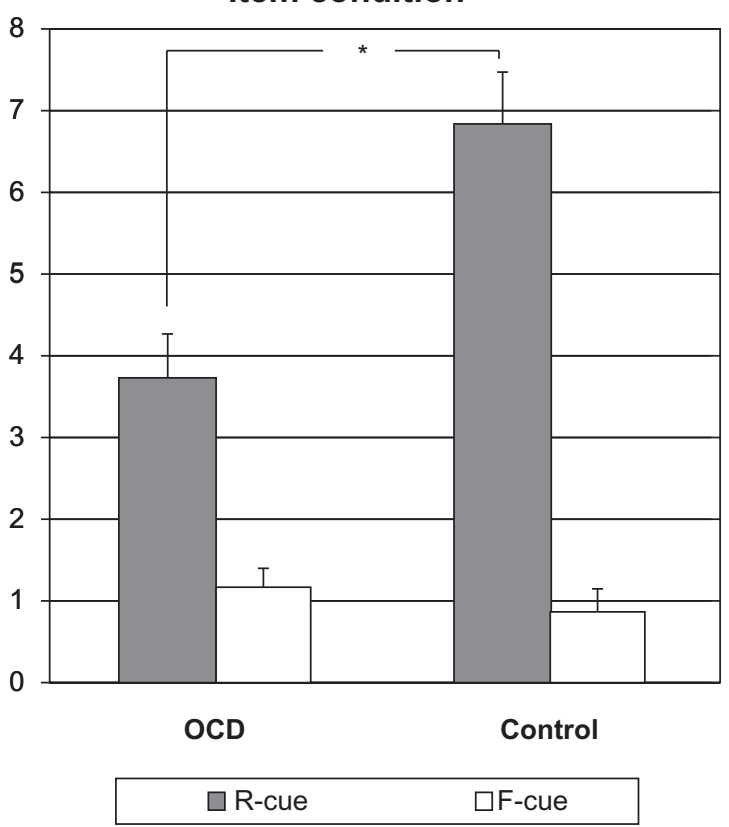

Figure 2 Mean numbers of correct words recalled under the List condition (left panel) and Item condition (right panel). Filled columns represent numbers of remember (R)cued words correctly recalled, while open columns represent numbers of forget $(F)$-cued words correctly recalled. Vertical bars represent the standard error of the mean.

numbers of R-cued words recalled by OCD patients (List, 4.75; Item, 3.75) were significantly less than those recalled by healthy controls (List, 6.76; Item, 6.88). On the other hand, OCD patients $(2.89,1.18)$ and healthy controls $(3.05$, 0.86 ) recalled similar numbers of F-cued words under either condition. No other interaction was found to be significant.

Two-way ANOVA (Group $\times$ Task condition) indicated that the only significant main effect was Group $(\mathrm{F}[1,42]=17.59$, $P<0.05)$. OCD patients exhibited significantly smaller directed forgetting effects than did healthy controls under both List (1.9 $\pm 2.4,4.4 \pm 3.1)$ and Item (2.6 $\pm 3.1,5.3 \pm 2.9)$ conditions, respectively. No interaction was significant.

\section{Discussion}

In this experiment, we have first confirmed that healthy subjects demonstrate significant increment in the recall of F-cued words only when tested with a list-method directed forgetting paradigm, as opposed to the recall of R-cued words that are unaffected by task methods (list- and itemmethods), as have been previously reported in a number of studies. ${ }^{22,23}$ This increment in the recall of F-cued words in List condition compared with Item condition supported a conditional distinction. Moreover, OCD patients performed in an analogous fashion, and so it is reasonable to propose that our materials and methods in a directed forgetting paradigm were equally reliable and valid to healthy persons and OCD patients.
In our directed forgetting paradigm, OCD patients recalled significantly fewer R-cued words than control participants under either List and Item conditions, while they recalled about as many F-cued words as control participants. In List condition, although OCD patients did inhibit retrieval of F-cued words as control participants did, they could not recall as many R-cued words as control participants did. In the same way, OCD patients recalled fewer R-cued words than control participants, because they encoded the same number of F-cued words as control participants in the Item method. In other words, OCD patients did recall F-cued words, and the encoding and retrieval of F-cued words accordingly blocked the recall of R-cued words. Consequently, OCD patients showed significantly smaller directed forgetting effects than control participants under both of the two separate conditions.

In the List method, R-cued and F-cued words are subjected to an identical encoding process, so the directed forgetting effect is believed to reflect successful retrieval inhibition or the intentional suppression of F-cued words. In the Item method, presentation of each word is followed by either a "remember" or "forget" instruction, so selective encoding or selective rehearsal is considered the key factor underlying the directed forgetting effect. The results of this study suggested that both encoding and retrieval processes in OCD patients differed from those in control participants, causing inferior memory performance in OCD patients. 
In the directed forgetting paradigm, participants were given an excessive number of words to learn $(n=24$ in the present study), and then were required to remember as many necessary (R-cued) words as possible following instructions to intentionally forget unnecessary (F-cued) words. However, although OCD patients recalled as many unnecessary (F-cued) words as control participants, they were less able to recall necessary (R-cued) words. Thus, OCD patients showed impaired selective recall of necessary words because incidental remembering of unnecessary words intruded. OCD patients appeared to try to remember and recall as many words as possible irrespective of R-cued or F-cued status, and so it resulted in relatively poorer recall of necessary words than of unnecessary words.

Using the same directed forgetting paradigm, it has been reported that OCD patients showed a decreased directed forgetting effect only for the stimulus words that they considered negative ${ }^{24}$ and a smaller directed forgetting effect for words with negative valence than for neutral words. ${ }^{26}$ The results of these studies suggest that OCD is characterized by an intentional cognitive inhibition deficit, which is specific to inhibition of retrieval of negative information. Our results in the List method results similarly suggested reduced retrieval inhibition of unnecessary (F-cued) words in OCD patients compared to control participants, but our findings were broader. Our OCD patients showed reduced retrieval inhibition of neutral information in our paradigm. Thus, our OCD patients exhibited reduced retrieval inhibition of irrelevant material regardless of emotional significance when given various items to learn and to organize in memory.

Such reduced retrieval inhibition could be viewed as frontal dysfunction. ${ }^{26}$ Mimura et al examined patients with frontal lobe damage using the same directed forgetting paradigm. ${ }^{32}$ Their frontal lobe damage patients performed well under the Item condition, but under the List condition they recalled fewer R-cued words and more F-cued words than patients with temporoparietal damage. This indicated impairment of an inhibitory process at the retrieval stage.

Our OCD patients showed difficulties in selective encoding in addition to reduced retrieval inhibition. Under the Item condition, OCD patients recalled as many unnecessary (F-cued) words as control participants, while they recalled fewer necessary (R-cued) words. In this sense, difficulties of OCD patients in directed forgetting appeared qualitatively different from those of patients with frontal lobe damage. However, another line of reasoning may suggest that impairment in selective encoding is also a consequence of frontal dysfunction. Deficits in selective encoding of necessary words may well involve poor organizational strategies caused by frontal dysfunction. Although patients with frontal lobe damage examined by Mimura et al were unimpaired under the Item condition, other studies indicated deficient encoding strategies in patients with frontal dysfunction. ${ }^{16-20}$ Such neuropsychological studies suggesting that OCD patients show decreased recall performance in memory tasks favor deficient frontal-related organizational strategies rather than a memory deficit per se.

Purcell et al reported that specific cognitive deficits of OCD patients related to spatial working memory and visual memory were qualitatively similar to those of patients with frontal lobe or subcortical damage. ${ }^{33}$ Such frontal-striatal system dysfunction has been implicated in neurobiological models of OCD. ${ }^{34-36}$ In addition, a recent neuroimaging study of a directed forgetting paradigm demonstrated that intentional forgetting is an active process requiring additional neural structures involving intentional remembering and unintentional forgetting, and that frontal control processes may be critical for these functions. ${ }^{37}$ In the present study, OCD patients were not impaired in any of three tests of frontal-executive function (the Modified Stroop Test, Word List Generation, and the Wisconsin Card Sorting Test). Dissociation of functional focus between such frontalexecutive tests and OCD pathology may partially account for our failure to directly demonstrate frontal-lobe dysfunction. Future studies including both the directed forgetting paradigm and functional neuroimaging in OCD are warranted.

Although we demonstrated selective impairment of episodic memory in OCD, there are some limitations in our study. The major one is the small number of participants, the imbalance in sample size, and the age range between the OCD and control groups. The standard deviation for the age of OCD patients was comparatively larger than that for the control group. Furthermore, although the OCD group was neurocognitively comparable to our database of healthy persons matched in age, it remained uncertain whether the OCD group was comparable to the control group. However, as previous literature consistently indicated preserved general intelligence, language, and verbal memory abilities among OCD patients, ${ }^{9-14}$ it is important to note that OCD patients performed poorly in difficult memory tasks such as this directed forgetting paradigm, although OCD patients did perform conventional word list learning (Ray AuditoryVerbal Learning Test) equivalently to healthy controls. Another limitation is the lack of control with regard to the level of education between OCD and control groups. The results of our study could simply be explained by educational 
differences. Fourth, while healthy volunteers were free of any medications, all of our OCD patients were on medication at the time of evaluation. Medication may influence memory performance, although there have been some reports that the medication status of OCD patients was unrelated to neuropsychological performance. ${ }^{38,39}$ In order to eliminate the effect of medication, it is desirable to replicate the experiment with drug-free patients in the future. Fifth, we did not systematically assess the scale of depression in OCD patients. OCD patients with a comorbid depressive disorder were excluded in current study, but comorbid depressive symptoms could have an effect on memory performance in $\mathrm{OCD}^{5,40}$ and so should have been considered. Moreover, we did not systematically assess the dimension of symptoms in OCD patients. Since the symptom dimensions in OCD were associated with neuropsychological profiles including the assessment of episodic memory, attention, and executive functioning, ${ }^{41}$ it would be more beneficial if we examined associations between clinical dimensions and task performance in OCD patients. Finally, it is possible to assume that the reported difference between R-cued and F-cued words in OCD could reflect differences in memory confidence and meta-memory, ${ }^{1-4,15}$ but a measure of memory confidence was not implemented in our study. We hope to explore this issue in future research.

\section{Conclusion}

This is the first study examining episodic memory in OCD patients with both a list- and item-method directed forgetting paradigm, and we demonstrated that OCD patients showed unique patterns of remembering and forgetting processes in learning neutral nouns, associated with reduced directed forgetting effect. The results of our study showed both reduced retrieval inhibition and inefficient encoding strategies in OCD patients. Both of these observations may support neurobiological models proposing frontal-subcortical system dysfunction in OCD. This memory problem in OCD patients did not involve inability to recall words to be remembered, but rather intentional remembering of unnecessary events that interfered with recall of necessary events. We emphasize that OCD patients remember every material regardless of its necessity or importance, and accordingly that unfavorable memory intrudes necessary, important and favorable items to remember. As OCD patients are less likely to delete unfavorable memories, their ongoing episodic memory would be affected. Such deficits specific to OCD contribute to a vicious cycle involving chronic doubt, intrusive thoughts and repetitive behavior.

\section{Acknowledgments}

This work was supported in part by a Grant-in-Aid from the Ministry of Education, Culture, Sports, Science, and Technology of Japan (MM) and a Showa University Grant-in-Aid for Innovative Collaborative Research Projects (MM).

\section{Author's contributions}

MK carried out the experiment, performed the statistical analysis, and drafted the manuscript. SN and SK participated in the design of the study. KS and MM recruited OCD patients, and MM helped to draft the manuscript. All authors read and approved the final manuscript.

\section{Disclosure}

The authors declare no conflicts of interest in this work.

\section{References}

1. Zitterl W, Urban C, Linzmaryer L, et al. Memory deficits in patients with DSM-IV obsessive-compulsive disorder. Psychopathology. 2001;34 113-117.

2. van den Hout M, Kindt M. Repeated checking causes memory distrust Behav Res Ther. 2003,41:301-316.

3. van den Hout M, Kindt M. Phenomenological validity of an OCDmemory model and the remember/know distinction. Behav Res Ther. 2003;41:369-378.

4. Tuna S, Tekcan AI, Topcuoglu V. Memory and metamemory in obsessive-compulsive disorder. Behav Res Ther. 2005;43:15-27.

5. Moritz S, Birkner C, Kloss M, et al. Impact of comorbid depressive symptoms on neuropsychological performance in obsessive-compulsive disorder. J Abnorm Psychol. 2001;110:653-657.

6. Tallis F. The neuropsychology of obsessive-compulsive disorder: A review and consideration of clinical implications. Br J Clin Psychol. 1997;36:3-20.

7. Greisberg S, McKay D. Neuropsychology of obsessive-compulsive disorder: A review and treatment implications. Clin Psychol Rev. 2003; 23:95-117.

8. Muller J, Robert JE. Memory and attention in obsessive-compulsive disorder: a review. J Anxiety Disord. 2005;19:1-28.

9. Boone KB, Ananth J, Philpott L, Kaur A, Djenderedjian A. Neuropsychological characteristics of nondepressed adults with obsessive-compulsive disorder. Neuropsychiatry Neuropsychol Behav Neurol. 1991;4:96-109.

10. Zielinski CM, Taylor MA, Juzwin KR. Neuropsychological deficits in obsessive-compulsive disorder. Neuropsychiatry Neuropsychol Behav Neurol. 1991;4:110-126.

11. Christensen KJ, Kim SW, Dysken MW, Hoover KM. Neuropsychological performance in obsessive-compulsive disorder. Biol Psychiatry. 1992;31:4-18

12. Dirson S, Bouvard M, Cottraux J, Martin R. Visual memory impairment in patients with obsessive-compulsive disorder: A controlled study. Psychother Psychosom. 1995;63:22-31.

13. Cohen LJ, Hollander E, DeCaria CM, et al. Specificity of neuropsychological impairment in obsessive-compulsive disorder: a comparison with social phobic and normal control subjects. J Neuropsychiatry Clin Neurosci. 1996;8:82-85.

14. Savage CR, Keuthen NJ, Jenike MA, et al. Recall and recognition memory in obsessive-compulsive disorder. $J$ Neuropsychiatry Clin Neurosci. 1996;8:99-103.

15. Moritz S, Jacobsen D, Willenborg B, Jelinek L, Fricke S. A check on the memory deficit hypothesis of obsessive-compulsive checking. Eur Arch Psychiatry Clin Neurosci. 2006;256:82-86. 
16. Savage CR, Baer L, Keuthen NJ, Brown HD, Rauch SL, Jenike MA. Organizational strateties mediate nonverbal memory impairment in obsessive-compulsive disorder. Biol Psychiatry. 1999;45:905-916.

17. Savage CR, Deckersbach T, Wilhelm S, et al. Strategic processing and episodic memory impairment in obsessive compulsive disorder. Neuropsychology. 2000;14:141-151.

18. Deckersbach T, Otto MW, Savage CR, Baer L, Jenike MA. The relationship between semantic organization and memory in obsessivecompulsive disorder. Psychother Psychosom. 2000;69:101-107.

19. Cabrera AR, McNally RJ, Savage CR. Missing the forest for the trees? Deficient memory for linguistic gist in obsessive-compulsive disorder. Psychol Med. 2001;31:1089-1094.

20. Savage CR, Deckersbach T, Heckers S, et al. Prefrontal regions supporting spontaneous and directed application of verbal learning strategies. Evidence from PET. Brain. 2001;124:219-231.

21. MacLeod CM. Directed forgetting affects both direct and indirect tests of memory. J Exp Psychl Learn Mem Cogn. 1989;15:13-21.

22. Basden BH, Basden DR, Gargano G. Directed forgetting in implicit and explicit memory test: A comparison of methods. J Exp Psychl Learn Mem Cogn. 1993;19:603-616.

23. MacLeod CM. The item and list methods of directed forgetting: test differences and the role of demand characteristics. Psychon Bull Rev. 1999;6:123-129.

24. Wilhelm S, McNally RJ, Baer L, Florin I. Directed forgetting in obsessive-compulsive disorder. Behav Res Ther. 1996;34:633-641.

25. Tolin DF, Hamlin C, Foa EB. Directed forgetting in obsessive-compulsive disorder: replication and extension. Behav Res Ther. 2002;40:793-803.

26. Bohne A, Keuthen NJ, Tuschen-Caffier B, Wilhelm S. Cognitive inhibition in trichotillomania and obsessive-compulsive disorder. Behav Res Ther. 2005;43:923-942.

27. American Psychiatric Association. Diagnostic and Statistical Manual of Mental Disorders. 4th ed. Washington, DC: American Psychiatric Association Press; 1944.

28. First MB, Spitzer RL, Gibbon M, Williams JB. Structured Clinical Interview for DSM-IV. Clinical Version (SCID/CV). Washington, DC: American Psychiatric Association Press; 1997.

29. Goodman WK, Prince LH, Rasmussen SA, et al. The Yale-Brown Obsessive Compulsive Scale. I. Development, use and reliability. Arch Gen Psychiatry. 1989;46:1006-1011.
30. Bollini P, Pampallona S, Tibaldi G, Kupelnick B, Munizza C. Effectiveness of antidepressants: Meta-analysis of dose-effect relationships in randomised clinical trials. Br J Psychiatry. 1999;174: 297-303.

31. Sakuma N, Ijuin M, Fushimi T, et al. NTT database series Nihongo-no Goitokusei (Lexical properties of Japanese). Tokyo, Japan: Sanseido; 2005 .

32. Mimura M, Komatsu S, Kato M, Umeda S, Saito F, Kahima H. Directed forgetting effect in patients with frontal lobe damage. Brain Cogn. 2001;47:343-346.

33. Purcell R, Maruff P, Kyrios M, Pantelis C. Cognitive deficits in obsessive-compulsive disorder on tests of frontal-striatal function. Biol Psychiatry. 1998;43:348-357.

34. Baxter LR, Schwartz JM, Bergman KS, et al. Caudate glucose metabolic rate changes with both drug and behavior therapy for obsessivecompulsive disorder. Arch Gen Psychiatry. 1992;49:681-689.

35. Rauch SL, Jenike MA, Alpert NM, et al. Regional cerebral blood flow measured during symptom provocation using oxygen 15-labeled carbon dioxide and positron emission tomography. Arch Gen Psychiatry. 1994;51:62-70.

36. Saxena S, Brody AL, Schwartz JM, Baxter LR. Neuroimaging and frontal-subcortical circuitry in obsessive-compulsive disorder. Br J Clin Psychiatry Suppl. 1998;35:26-37.

37. Wylie GR, Foxe JJ, Taylor TL. Forgetting as an active process: An fMRI investigation of item-method-directed forgetting. Cereb Cortex. 2008;18:670-682.

38. Mataix-Cols D, Alonso P, Pifarré J, Menchón JM, Vallejo J. Neuropsychological performance in medicated vs. unmedicated patients with obsessive-compulsive disorder. Psychiatry Res. 2002;109: 255-264

39. Simpson HB, Rosen W, Huppert JD, Lin SH, Foa EB, Leibowitz MR. Are there reliable neuropsychological deficits in obsessive-compulsive disorder? J Psychiatr Res. 2006;40:247-257.

40. Basso MR, Bornstein RA, Carona F, et al. Depression accounts for executive function deficits in obsessive-compulsive disorder. Neuropsychiatry Neuropsychol Behav Neurol. 2001;14:241-245.

41. Hashimoto N, Nakaaki S, Omori IM, et al. Distinct neuropsychological profiles of three major symptom dimensions in obsessive-compulsive disorder. Psychiatry Res. 2011;187:166-173.
Neuropsychiatric Disease and Treatment

\section{Publish your work in this journal}

Neuropsychiatric Disease and Treatment is an international, peerreviewed journal of clinical therapeutics and pharmacology focusing on concise rapid reporting of clinical or pre-clinical studies on a range of neuropsychiatric and neurological disorders. This journal is indexed on PubMed Central, the 'PsycINFO' database and CAS, and is the official

\section{Dovepress}

journal of The International Neuropsychiatric Association (INA). The manuscript management system is completely online and includes a very quick and fair peer-review system, which is all easy to use. Visit http://www.dovepress.com/testimonials.php to read real quotes from published authors. 